九州大学学術情報リポジトリ

Kyushu University Institutional Repository

\title{
Numerical Simulation of Shiroishi Area Aquifer
}

Shaikh, Muhammad Arab

Laboratory of Irrigation Engineering, Faculty of Agriculture, Kyushu University

Tanabe, Kunimi

Laboratory of Irrigation Engineering, Faculty of Agriculture, Kyushu University

https://doi.org/10.5109/22865

出版情報 : 九州大学大学院農学研究院紀要. 19 (1)，pp.11-31，1974-10. Kyushu University バージョン：

権利関係 : 
J. Fac. Agr., Kyushu Univ., 19, 11-31 (1974)

\title{
Numerical Simulation of Shiroishi Area Aquifer
}

\author{
Muhammad Arab Shaikh and Kunimi Tanabe \\ Laboratory of Irrigation Engineering, Faculty of Agriculture, \\ Kyushu University, Fukuoka
}

(Received July 2, 1974)

\begin{abstract}
A mathematical model solved by ADIP method is used to study the dynamic behavior of Shiroishi area aquifer, Saga Prefecture, Kyushu, Japan, in response to pumping from irrigation and municipal wells, where subsidence has been resulted due to heavy pumping.

The two dimensional model with constant hydraulic head boundaries (where the head has not been changed) were used to compute the seasonal drawdowns and these computed drawdowns were compared with field data.

The drawdown contours of September were found in good agreement with field data, but of February showed low drawdowns. It was thought that the irrigation period is from May to September, so the recharge was cut off after September and in doing this way the computed and field contours were found in good agreement, thus the recharge occurs in the irrigation period only.

In this study the qualitative relationships among drawdown, subsidence and thickness of clay layer were determined and it was concluded in the following way: For same drawdown, the subsidence is higher for thicker clay layer, and for constant thickness, the subsidence increases with increase in drawdown.
\end{abstract}

\section{INTRODUCTION}

Ground water has been utilized for irrigation purpose for thousands of years, but nowadays it has become one of main resources for municipal and industrial water supply also along with irrigation.

The ground water has following advantages over the surface water ; (a) Constant temperature, (b) Slow movement, (c) Good quality, (d) Greatly reduced evaporation losses. These advantages are of particular value in arid and semiarid regions, where annual evaporation from surface reservoirs may exceed 6 or 7 acre-ft per acre.

The development of powerful high speed drilling equipments and the innovation of submerge pumps have accelerated the use of ground water which has caused uncontrolled exploitation of it, and has resulted in various socioeconomic problems. Among these problems, the heavy drawdown of water levels in the aquifer, subsidence and sea water intrusion are mostly common. In order to find out solutions of these problems, it is necessary to understand the dynamic behavior of the ground water in the aquifer. For this purpose the mathematical models have proved very valuable tools for studying the dynamic behavior. In mathematical models the essential characteristics of a basin are 
reproduced in a model which is then studied in the abbreviated time scale.

It is well agreed that water level decline is the one of main causes for occurrence of other two problems (subsidence and sea water intrusion), so here ADIP method is used to compute the regional hydraulic head drawdowns in the Shiroishi Area aquifer, Saga Prefecture, Kyushu, in response to seasonal pumping, where subsidence has been resulted due to heavy pumping.

\section{GEOLOGY AND HYDROLOGY}

The Shiroishi area borders on the Ariake sea, an inland sea in the North Western Kyushu (Fig. 1). The concerned area lies on a delta which has been formed by Rokkaku river. It occupies an area of about 8800 ha, $70 \%$ of which has been reclaimed since the 13 th century and $80 \%$ is under cultivation at present, mostly paddy fields, There are 220 wells out of which 100 are for irrigation purpose which command 2800 ha of paddy fields and the remaining are for municipal supply. The location of these wells is shown in Fig. 2.

The water resources for irrigation in the area have been rivers, ponds and creeks, since 1925 tube wells have been introduced into the area for utilizing ground water for irrigation and municipal water supply. The number of tube wells has increased year by year, but a drought year in 1955 accelerated sinking of tube wells.

The bed layer of the area is made up of practically all Pleistocene which consists of sand and gravel layers and pyroclastic layers. The main aquifer is divided into two zones. The lower sand and gravel facies of the formation form champion aquifers in the area. The facies develop more than $200 \mathrm{~m}$ thick from a depth of arround $30 \mathrm{~m}$. The upper Pleistocene formation overlies it and a thin volcanic ash layer lies in between. The formation consists mainly of silt to coarse sand and forms a confined aquifer. The main aquifer consists of high and semi-permeable layers, but in a mathematical simulation an idealization of the aquifer into a material of uniform hydraulic characterstics is taken into account.

The top formation in the area is composed of recent clay with soft and stick

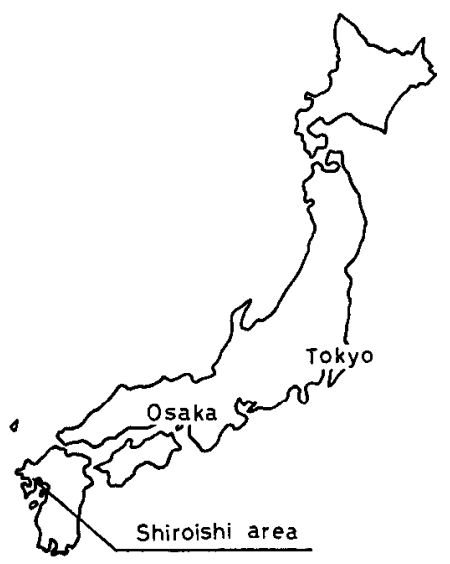

Fig. 1. Location of Shiroishi area. 


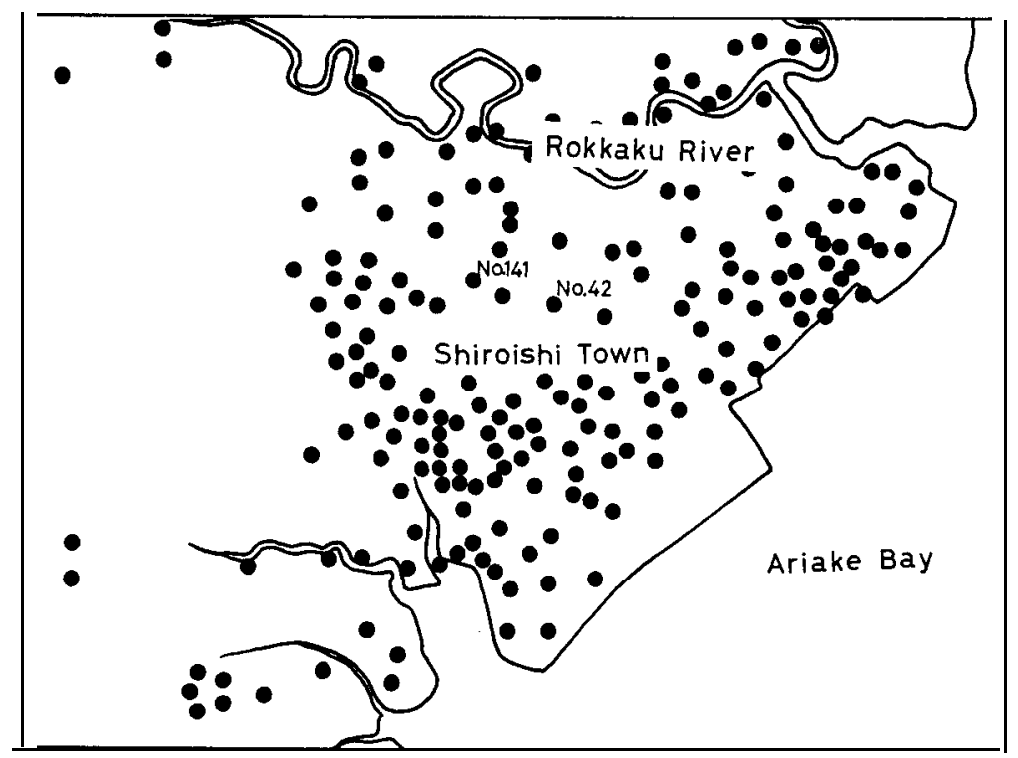

Fig. 2. Distribution of wells.

properties called Ariake clay; which is also considered one of main causes of subsidence. The thickness of Ariake clay varies from place to place and is about $20 \mathrm{~m}$ thick averagely (Fig. 3).
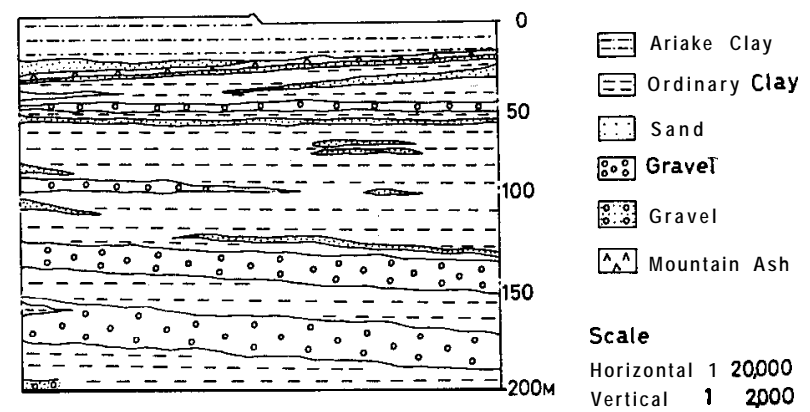

Fig. 3. Geological description.

\section{THEORETICAL ANALYSIS}

\section{Darcy's law}

The flow of water downward through vertical filter beds was investigated by French hydraulic engineer Darcy in 1856, that is now known as Darcy's law, governing the flow of water through porous media and is given by following equation.

$$
\mathrm{Q}=K \cdot A\left(H_{1}-H_{2}\right) / L
$$




$$
v=Q / A=K\left(H_{1}-H_{2}\right) / L
$$

By considering the head loss $\left(H_{1}-H_{2}\right)$ is uniform in the direction of flow, then above equation in the generalized or in gradient form becomes as follows:

$$
v=-K \frac{d h}{d s}
$$

Minus sign shows that head is decreasing in the direction of flow. $K$ is permeability co-efficient or transmission co-efficient $(\boldsymbol{L} / T)$.

$$
K=c d^{2} \frac{\gamma}{\mu}=k \frac{\gamma}{\mu}
$$

where cd" characterizes the properties of the medium, $\boldsymbol{k}$ is intrinsic permeability depending on properties of porous media $\left(\mathrm{L}^{2}\right) \cdot \gamma$ and $\mu$ are properties of the liquid flowing through porous media.

In case of different permeabilities in different directions the components of velocity in rectangular co-ordinates when fluid is considered homogeneous are as follows :

$$
v_{x}=-K_{x} \frac{\partial h}{\partial x}, \quad v_{y}=-K_{y} \frac{\partial h}{\partial y}, \quad v_{z}=-K_{z} \frac{\partial h}{\partial z}
$$

In case of isotropic medium $K_{x}=K_{y}=K_{z}$.

\section{Flow in an elastic aquifer}

The differential equation governing the flow of water in an elastic aquifer was derived by Jacob (1940). While accounting for the conservation of fluid mass in a control volume, it was first considered non-deforming to calculate the net inward mass flux and then was allowed to deform to compute the rate of change of fluid mass in the volume. Cooper (1966) derived the same equation by considering vertical co-ordinate $\mathrm{z}$ only as deforming co-ordinate, so it is still considered valid with certain approximation.

\section{Derivation of differential equation}

For ground water flow the conservation of mass principle requires that the rate of increase or decrease of fluid mass in an element situated in the flow field must be equal to the difference between the rate of influx and efflux, i. e,

Mass inflow rate=Mass outflow rate + change of mass storage.

Consider an elemental parallelpipedum of porous medium completly saturated with fluid density (Fig. 4).

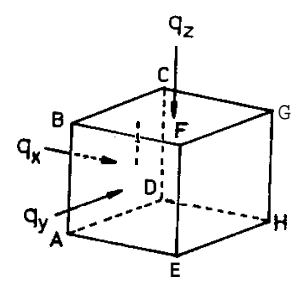

Fig. 4. Elemental Parallelpipedum. 
The mass inflow rate through face $\mathrm{ABCD}=\rho q_{x}$ dy $d z$ where $q_{x}$ is the specific discharge normal to the area $A A$ in the direction $\mathrm{X}$ and $p$ is fluid density. Mass outflow rate through face $\mathrm{EFGH}=\left\{\rho q_{x}+\frac{\partial}{\partial x}\left(\rho q_{x}\right) d x\right\} y d z$. Thus net inward flux $=-\frac{\partial}{\partial x}$ $\left(\rho q_{x}\right) d x d y d z$. Making similar equations for sides ABEF, DCGH and ADHE, BCGF and adding these results we have net inward flux $=-\left\{\frac{\partial}{\partial x}\left(\rho q_{x}\right)+\frac{\partial}{\partial y}\right.$ $\left.\left(\rho q_{y}\right)+\frac{\partial}{\partial z}\left(\rho q_{z}\right)\right\} d x d y d z$. This must be equal to change of mass storage in a given time. The mass of fluid $\boldsymbol{M}$ stored in the element is,

$$
\Delta M=n \rho A x A y A z
$$

where $\mathrm{n}$ is porosity of the porous medium.

In the consideration of the change of $\boldsymbol{M}$ in time, $\boldsymbol{n}$ and $\boldsymbol{z}$ may vary due to vertical compression or expansion of the medium and may change in time as well as in place. But the variation of lateral dimensions of the aquifer is considered negligible because of constraints of it by their surroundings, therefore the differential equation will be of the following form,

$$
\frac{\partial(\Delta M)}{\mathrm{at}}=\left(n \rho \frac{\mathrm{\partial}(\Delta z)}{\partial t}+\rho \Delta z \frac{\partial n}{\partial t}+\Delta z n \frac{\partial \rho}{\partial t}\right) A x A y
$$

Now these three terms will be expressed in terms of the compressibility $\alpha$ of the aquifer, the compressibility $\beta$ of the fluid and the pore-water pressure.

The vertical compressibility of the granular skeleton of the medium is expressed as $\alpha=1 / E_{s}$, where $E_{s}$ is bulk modulus of elasticity of the skeleton. The stress $\sigma$ on the intergranular skeleton in the vertical direction is called inter-granular pressure or effective stress.

$$
\begin{aligned}
& E_{\mathrm{s}}=\frac{\Delta \text { stress }}{\Delta \mathrm{strain}}=\frac{d \sigma}{-d(\Delta z) / \Delta z} \\
& n \rho \frac{\mathrm{a} A Z}{\mathrm{at}}=-\alpha n \rho \Delta z \frac{\partial \sigma}{\partial t}
\end{aligned}
$$

The volume of solid grains $V_{s}=(1-n) \Delta x A y A z$ is constant because the compressibility of the individual grains is considerably smaller than that of their skeleton, and is also smaller than compressibility of water, therefore total derivative will be zero.

$$
d\left(\Delta V_{s}\right)=d\{(1-n) \Delta x \quad A y \Delta z\}=0
$$

$A \boldsymbol{x}$ and $\boldsymbol{A y}$ in derivative are considered constants.

$$
\begin{aligned}
& \Delta z d(1-n)+(1-n) d(A z)=O \\
& \rho \Delta z \frac{\partial n}{\partial t}=-\rho \Delta z \alpha(1-n) \frac{\partial \sigma}{\partial t}
\end{aligned}
$$

Compressility $\boldsymbol{\beta}$ of the fluid is reciprocal of it's bulk modulus of elasticity, 


$$
\beta=-\frac{d(\Delta V) / \Delta V}{d P}
$$

The equation of conservation of mass is, $\rho \Delta V=\rho_{0} \Delta V_{0}=$ const. $\rho_{0}, \Delta V_{0}$ are constant reference values of density and elemental volume of the fluid. By differentiation it gives

$$
\rho \beta \frac{\partial P}{\partial t}=\frac{\partial \rho}{\partial t}
$$

where $P$ is pore-water pressure or neutral stress. At any depth intergranular pressure and pore-water pressure add to render the combined pressure and is constant with time,

$$
d \sigma=-d P
$$

Now adding these three quantities we have,

$$
\frac{\partial}{\partial t}(\Delta M)=\{n \rho \alpha+\rho(1-n) \alpha+n \rho \beta\} \Delta x \Delta y \Delta z \frac{\partial P}{\partial t}
$$

now equating the inward flux with change of mass storage,

$$
-\left\{\frac{\partial\left(\rho q_{x}\right)}{\mathrm{aX}}+\frac{\partial\left(\rho q_{y}\right)}{\partial y}+\frac{\partial\left(\rho q_{z}\right)}{\partial z}\right\}=\rho(\alpha+n \beta) \frac{\partial P}{\partial t}
$$

By considering density $p$ is constant then we have,

$$
-\left\{\frac{\partial q_{x}}{\partial x}+\frac{\partial q_{y}}{\partial y}+\frac{\partial q_{z}}{\partial z}\right\}=(\alpha+n \beta) \frac{\partial P}{\partial t}
$$

using the values of $q_{x}, q_{y}, q_{z}$ and $P=\gamma h=\rho g h$

$$
K\left(\frac{\partial^{2} h}{\partial x^{2}}+\frac{\partial^{2} h}{\partial y^{2}}+\frac{\partial^{2} h}{\partial z^{2}}\right)=\rho g(\alpha+n \beta) \frac{\partial h}{\partial t}
$$

Finally it becomes

$$
\begin{gathered}
\nabla^{2} h=S_{s} / K \frac{\partial h}{\partial t} \\
S_{s}=\rho g(\alpha+n \beta)
\end{gathered}
$$

where $\mathrm{g}$ is the acceleration of gravity, $S_{s}$ is specific storage with dimension $1 / \mathrm{L}$ and is defined the volume of water that a unit aquifer releases from storage because of expansion of water and compression of the grains under a unit decline in average head within the unit volume.

In the case of a confined aquifer of thickness $b$, the storage co-efficient $\mathrm{S}$ is defined, $S=S_{s} b . S$ is dimensionless and is defined as the amount of water in storage released from a column of aquifer with unit cross section under a unit decline of head, and transmissivity $T$ of the aquifer is as following,

$$
T=K b\left(\mathrm{~L}^{2} / \mathrm{T}\right) \text {. }
$$

$S$ and $T$ are called formation constants of the confined aquifer. $S_{\mathrm{s}} / K=S / T$, so in the case of confined aquifer the basic equation will become of the following 
form,

$$
\nabla^{2} h=\frac{S}{T} \frac{\partial h}{\partial t}
$$

For two dimensional flow in a confined aquifer of uniform thickness the equation will be in the following form,

$$
\frac{\partial^{2} h}{\partial x^{2}}+\frac{\partial^{2} h}{\partial y^{2}}=\frac{s}{T} \frac{\partial h}{\partial t}
$$

In the simple form of the equation, the transmissivity and storativity are shown constants for the given aquifer but in the filed are variable.

\section{Numerical model}

Finite difference approximation In the aquifer simulation it is necessary first of all to represent the aquifer mechanism with suitable differential equation, and then within the equation the true complexity of the aquifer system is embeded. In numerical solution the partial differential equation is replaced by it's corresponding finite difference equation, and by solving the resulting difference equation the head distribution in the aquifer is obtained. A convenient procedure which is used in formulating the difference equation is to start with a grid network which can be superimposed over a scaled map of the reservoir. The values of first and second differential equations in the form of finite difference are found in the following manner :

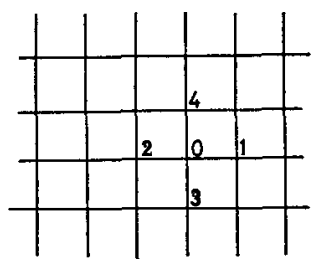

Fig. 5. Finite difference approximation.

$\boldsymbol{h}_{v}, h, h, \boldsymbol{h}_{3}$ and $\boldsymbol{h}_{4}$ are the heads at $0,1,2,3$, and 4 nodal points respectively, then values of derivatives are as follows:

$$
\begin{aligned}
& \left(\frac{\partial h}{\partial x}\right)_{0-1}=\frac{h_{1}-h_{0}}{A X-}, \quad\left(\frac{\partial h}{\partial y}\right)_{4-0}=\frac{h_{4}-h_{0}}{\Delta Y} \\
& \left(\frac{\partial h}{\partial x}\right)_{\text {2-o }} Y_{\mathscr{F}^{-} h_{2}}^{h^{\prime}}, \quad\left(\frac{\partial h}{\partial y}\right)_{\text {b-3 }}=\frac{h_{0}-h_{3}}{\Delta Y}
\end{aligned}
$$

Equations (4) are called forward difference approximation. Equations (5) are backward difference approximation. The second order derivatives are as rate of change of first order derivatives or as the difference between forward and backward differences divided by distance between two points.

$$
\left(\frac{\partial^{2} h}{\partial x^{2}}\right)_{0}=\frac{1}{\Delta X^{2}}\left(h_{1}+h_{2}-2 h_{0}\right)
$$




$$
\left(\frac{\partial^{2} h}{\partial y^{2}}\right)_{0}=\frac{1}{\Delta} Y^{2}\left(h_{3}+h_{4}-2 h_{0}\right)
$$

The head at the nodal point 0 is equal to the average of it's values along a circle around the point 0 .

Alternate direction implicit procedure The computational techniques employed to solve the finite difference approximations of the unsteady flow equations were developed originally by mathematicians for application to oil reservoirs and heat flow problems.

The first contribution in this field was attributed by Peaceman and Rachford (1955). Later, the California Department of Water Resources made the first attempt to evaluate numerically the response of ground water levels due to pumping (Tyson and Weber, 1964), McCarty (1958) used same method for calculation of pressure distribution in the oil reservoir, Bredehoeft and Pinder (1970) also used the same method for determining the response of aquifer due to pumping.

The methods used for solving finite difference equations are classified into two groups; (a) Explicit and (b) Implicit.

Explicit method is simple to solve but it requires an uneconomical a large number of time steps of limited size. In this method the correct heads for each node at various time steps are determined in terms of the known heads at adjacent nodes.

Implicit method have been successfully used, in this method there is no limit of the time step. For Shiroishi area also it is decided to use this method.

This is two step process involving two difference equations rather than the usual one. In the case where it is used to iterate the solution of the required equation then each step of iteration is considered as a time step of a corresponding unsteady problem.

In the first step, one of the second derivatives (say X-direction) be replaced with a second difference expressed in terms of the unknown values of $\boldsymbol{h}$, while the other second derivative term is to be evaluated interms of h's at the known time level and the equation is called implicit in the X-direction. The second step is performed in the similar manner reversing the positions of the second derivative terms with respect to known values in the $\mathrm{X}$-direction and unknown values in the Y-direction, this equation is implicit in the Y-direction.

The resulting difference equations are as follows:

$$
\begin{aligned}
& \left\{T_{i+1, j}\left(h_{i+1, j}-h_{i, j}\right)^{n+1}-T_{i-1, j}\left(h_{i, j}-h_{i-1, j}\right)^{n+1}\right\}+ \\
& \left\{T_{i, j+1}\left(h_{i, j+1}-h_{i, j}\right)^{n}-T_{i, j-1}\left(h_{i, j}-h_{i, j-1}\right)^{n}\right\} \\
= & S_{i, j} \frac{h_{i, j}^{n+1}-h_{i, j}^{n}}{\Delta T} . \Delta X \Delta Y+q_{i, j}^{n} \Delta X \Delta Y \\
& \left\{T_{i+1, j}\left(h_{i+1, j}-h_{i, j}\right)^{n+1}-T_{i-1, j}\left(h_{i, j}-h_{i-1, j}\right)^{n+1}\right\}+ \\
& \left\{T_{i, j+1}\left(h_{i, j+1}-h_{i, j}\right)^{n+2}-T_{i, j-1}\left(h_{i, j}-h_{i, j-1}\right)^{n+2}\right\} \\
= & S_{i} \frac{\left(h_{i, j}^{n+2}-h_{i, j}^{n+1}\right)}{A T} \Delta X \Delta Y+q_{i, j}^{n+1} \Delta X \Delta Y
\end{aligned}
$$


Equations (6) and (7) are implicit in $\mathbf{X}$ and $\mathbf{Y}$ directions respectively. In these equations the superscripts $n, n+1$ and $n+2$ refer to time levels and $q_{i, j}$ is the source or sink term to account for the effects of pumping or recharge per unit area, $\mathbf{i}$ and $\mathrm{j}$ denotes in the $\mathrm{X}$ and $\mathrm{Y}$ directions respectively. The calculation in the first step is successively carried on row by row till all the rows are completed, and in the second step the calculation is carried on column by column and in this way the computation is continued till the solution of the required period is achieved.

Boundary conditions After superimposing the grid network on the aquifer, then before analysis it is necessary to give the boundaries of the aquifer a physical meaning. If the boundaries of the aquifer are closed to the flow of fluids (impermeable boundaries), then these boundaries in the aquifer analysis can be simulated by assigning the values of permeabilities at all points in the grid network out side the limits of the aquifer equal to zero. If the influx of water across the particular portion of field boundaries is crossing then in this case, the appropriate constants or functions have to be introduced into the calculation in order to produce the magnitudes of that flow at these boundaries. In case of Shiroishi area, it was decided after analysing the actual data measured in the field of the various years, that it was found better to have constant head boundaries where head has not been changed for all time, so by drawing the constant head boundaries and assigning the zero drawdown on this boundary for all time, the data was analyzed. Recently in the same way, the radial hydraulic boundaries were assumed by Gambolati and Freeze (1973) while analyzing the subsidence at Venice. In Shiroishi area the grid network is adjusted in order to coincide with constant hydraulic boundaries as shown in the Fig. 6. The data of 1964-1965 and March 1966-March 1967 is analyzed here. The initial condition for the aquifer simulation is assumed zero drawn in the March 1964, and for year 1966-1967 the initial condition was drawn from field data, the data of year 19651966 was not available. The data of year 1964-1965 was used on the average basis but the data of the year 1966-1967 was used for each well pumped in the particular period.

Simplification of equations $f$ or computer program A pair of difference equations described in earlier paragraphs which apply equally to all interior and boundary points are written for each node along either a row or column in a series of
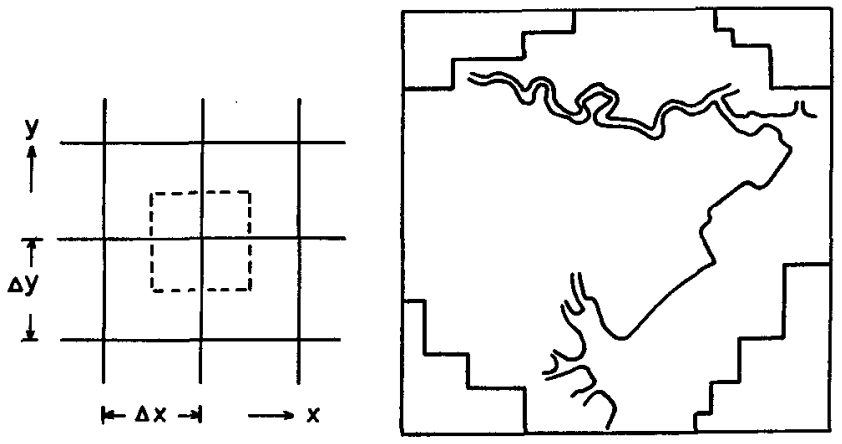

Fig. 6. Superimposed grid net. 
simultaneous equations, which are further simplified for drawdown computation inside the aquifer in the following way.

$$
\begin{aligned}
& \left\{\left(T_{i-1, j)} h_{i-1, j}-\left(T_{i-1, j}+T_{i+1 . j}+S_{i, j} \frac{\Delta X^{2}}{\Delta t}\right) h_{i, j}+\left(T_{i+1, j}\right) h_{i+1, j}\right\}^{n+1}\right. \\
& =q_{i, j} \Delta X^{2}-\left\{T_{i_{j} j-1} h_{i, j-1}-\left(T_{i, j-1}+T_{j_{. j+1}}-S_{i, j} \frac{\Delta X^{2}}{\Delta t}\right) h_{i, j}+T_{i, j+1 / 2} h_{i, j+1}\right\}^{u}
\end{aligned}
$$

By substituting the following expressions in above equation, finally we will get simple equation,

$$
\begin{aligned}
& A_{i}=T_{i-1, j} \\
& B_{i}=T_{i-1, j}+T_{i, j-1}+\frac{S_{i, j} \Delta X^{2}}{\Delta t} \\
& C_{i}=T_{i, j+1 / 2} \\
& D_{i}=q_{i, j} \Delta X^{2}-\left\{\left(T_{i, j-1}\right) h_{i, j-1}-\left(T_{i, j-1}+T_{i, j+1}-\frac{S_{i, j} \Delta X^{2}}{\Delta t}\right) h_{i, j}+T_{i, j+1} h_{i, j+1}\right\}^{n} \\
& \left(A_{i} h_{i-1, j}+B_{i} h_{i, j}+C_{i} h_{i+1, j}\right)^{n+1}=D_{i}
\end{aligned}
$$

This equation is implicit in the X-direction. This equation is written for all points along a typical row or column as the case may be, here consider the typical row with nodal points from 1 to $n$, then in this case points 1 and $n$ are on the boundary and as on the boundary head does not change as explained before, so the values of these points will not be calculated but are known at all times, so basic set of simultaneous equations for this row will be written in the following form from 2 to $n-1$ points,

$$
\begin{aligned}
A_{2} h_{1}+B_{2} h_{2}+C_{2} h_{3} & =D_{2} \\
A_{3} h_{2}+B_{3} h_{3}+C_{3} h_{4} & =D_{3} \\
A_{4} h_{3}+B_{4} h_{4}+C_{4} h_{5} & =D_{4}
\end{aligned}
$$

In these equations, the values of $\boldsymbol{h}$, and $\boldsymbol{h}_{n}$ are zero because boundary values are taken as reference values by considering the drawdown on the boundary equal to zero. The set of these equations are solved by using non-iterative technique and the resulting equations are as follows:

$$
\begin{array}{llrl}
h_{n-1}=G_{n-1} & h_{i}=G_{i}-C_{i} h_{i+1} / W_{i} & 2 \leqq i \leqq n-2 \\
W_{2}=B_{2} & W_{i}=B_{i}-A_{i} C_{i-1} / W_{i-1} & 3 \leqq i \leqq n-1 \\
G_{2}=D_{i} / W_{2} & G_{i}=\left(D_{i}-A_{i} G_{i-1}\right) / W_{i} & 3 \leqq i \leqq n-1
\end{array}
$$

the values of $\mathrm{G}$, and $W_{i}$ are computed in order of increasing $i$ followed by evaluation of $h_{i}$ in order of decreasing $i$. In this way the calculation is successively carried on row by row until all the rows are completed. The resulting values obtained by solving the simultaneous equations in the $\mathrm{X}$-implicit will be now 
initial conditions for the equations of Y-implicit direction. And similar computation is carried on column by column for next half time step. Thus in this way procedure will be continued till the forecast period is completed.

The clay layer overlying the main aquifer is called Ariake clay and it's depth varies from $14 \mathrm{~m}$ to $24 \mathrm{~m}$, this layer is treated here as semi-confining layer, and is linked with the main aquifer in the following way.

The replenishment of any aquifer is mainly from three sources and according to their contribution, $67 \%$ is from irrigation water, $30 \%$ streams and canals $3 \%$ rainfall. As already mentioned, the $80 \%$ of the concerned area is under paddy field cultivation, so main replenishment of the aquifer is considered irrigation water through the overlying clay layer. Bredehoeft and Pinder's model (1970) is used here by treating the flow in the main aquifer horizontal (two dimensional) and vertical in the clay layer. This model is effective because the permeability contrast is higher than two orders of magnitude.

The flux from the clay layer is calculated in the following way.

$$
\mathrm{Flux}=F(i, j)=-K_{i, j}\left(h_{i, j}-H_{i, j}\right) / L_{i, j}
$$

$L=$ Depth of the clay layer

$\mathrm{K}=$ Vertical hydraulic conductivity of the confining layer

$h_{i, 3}=$ Head in the main aquifer for certain point and known term (here draw. down level is considered.)

$H_{i,},=$ Head in the clay layer

Before the exploitation of the main aquifer is carried out, the water levels in the artesian aquifer (piezometric levels) are with in 1-2m from the ground surface, so here it is assumed that ground level and piezometric levels were coinciding with each other. As the water level in the main aquifer started to decline the water from the clay layer has been cause of replenishment of the aquifer, and it's rate should be proportional to head declination in the main aquifer. As the drawdown is measured from the ground surface, and head of irrigation water in the field is zero, so the term $\boldsymbol{H}_{\boldsymbol{i}, \boldsymbol{j}}$ in the equation is zero and the final equation is of the following form,

$$
F_{i, s}=-K_{i, j} h_{i, j} / L_{i, j}
$$

and then adding this term to the right side of the basic equation, it will become as follows.

$$
\left(A_{i} h_{i-1, j}+B_{i} h_{i, j}+C_{i} h_{i+1, j}\right)^{n+1}=D_{i}+K_{i, j} h_{i, j}^{n} / L_{i, j}
$$

\section{Input data}

In simulating the Shiroishi area aquifer, the area of the aquifer is discrete by two sets of orthogonal lines forming $27 \times 2$ '7 uniform meshes with sides of each mesh equal to $525 \times 525 \mathrm{~m}$ as shown in Fig. 6 . In this network each nodal point is considered as pumping center with number of wells grouped there or without any well, but treated as single well, wells lying within the mesh are shifted to nearest nodal point. The volumes of water pumped from each well in the aquifer were calculated by following equation, 


$$
\begin{array}{cc}
Q=\mathbb{P} n_{\mathrm{p}} / 0.222 H & \text { Ton } / \mathrm{min} \text {. (cubic meter } / \mathrm{min} \text { ) } \\
\mathbb{P}=\text { Horse power of motor } & \mathrm{Q}=\text { Pumped volume } \\
n_{p}=\text { Efficiency of the motor } & H=\text { Head of water (Depth of well) }
\end{array}
$$

The volumes calculated from each well are summed together and total volume per 10 days is calculated, because the time step of 10 days is considered in the program.

The actual pattern of pumpage for year 1964-1965 and 1966 - 1967 is shown in Fig. 7, and in this water pumped from municipal and irrigation wells is grouped together, and time unit is $\mathbf{1 0}$ days. The municipal wells have been pumped at constant volume per 10 days throughout the year, but irrigation wells have been used from June to Sept. only.
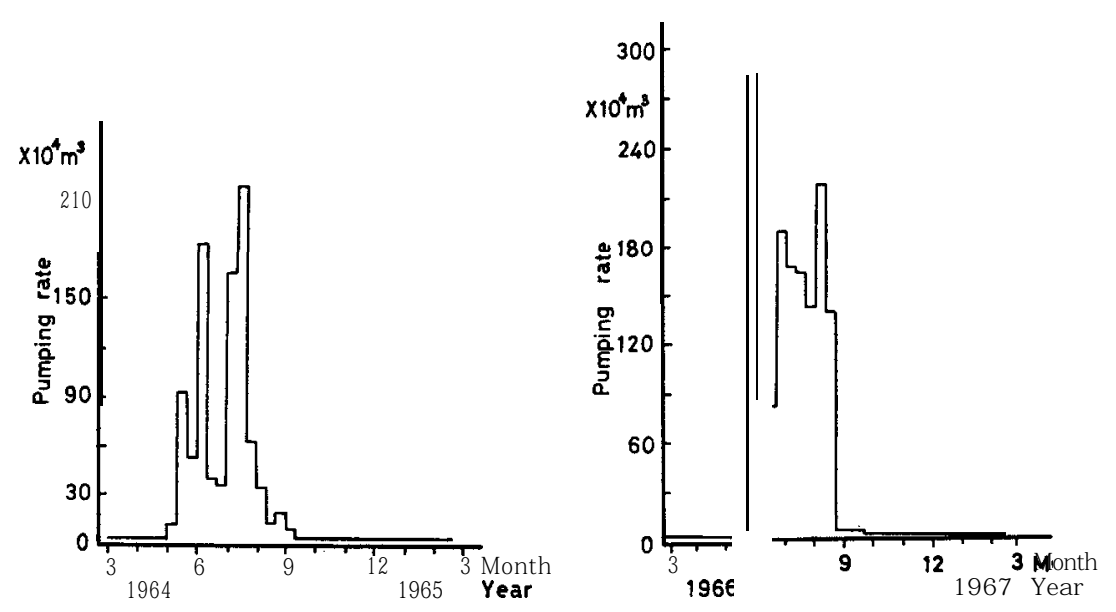

Fig. 7. Pumping pattern.

The total quantity pumped from all wells on the basis of above equation is $993274 \mathrm{~m}^{3} / 10$ days. In order to satisfy the ground water flow equation for unstead$y$ flow, the effects of changing rate of withdrawal are superimposed by multiplying $R(t)$ with $\mathrm{Q}$. The value of $\mathrm{Q}$ in the program is rate per day but drawdowns are calculated every 10 days. The value of $R(t)$ is calculated by dividing the actual pumped quantity shown in Fig. 7 for particular period with 993274 $\mathrm{m}^{3} / 10$ days and in this way $R(t)$ is the function of the time, by doing this way the actual pumped quantity is used in the program.

The co-efficient of storage as reported is between $1.42 \times 10^{-2}$ and $1.62 \times 10^{-2}$ (dimensionless), here it is decided to use the average of these two values, i. e. $S=1.5 \times 10^{-2}$.

The transmissivity of the aquifer varies with location but for homogeneous condition it is decided to use the average value of $T=3.0 \times 10^{-3} \mathrm{~m}^{2} / \mathrm{sec}$.

The value of the vertical hydraulic conductivity of the overlying clay layer is between $1.0 \times 10^{-6}$ and $1.75 \times 10^{-6} \mathrm{~cm} / \mathrm{sec}$, in order to find out the suitable value that was varied between these limits. 
The above stated value are described in the report of the Agriculture and Forestry Authority of Saga Prefecture (1967).

\section{RESULTS AND DISCUSSIONS}

The drawdowns obtained by using numerical model mentioned in earlier chapters are compared with field data, and the difference between them is discussed below,

(1) In order to find suitable model for Shiroishi area aquifer, the analysis was started first by considering the aquifer homogeneous and isotropic and average values of formation constants were used. The recharge from the confining layer was not considered in the first step, and the drawdown contours obtained with these assumptions for Sept., 1964 and Feb., 1965 are shown in Fig. 8. The field contours of the same year are shown in Fig. 9, by comparing computed and field contours, it can be easily concluded that computed drawdowns are not only higher but also their tendency is completly different.

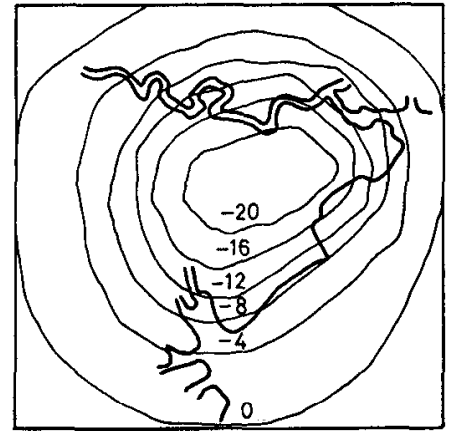

Sept : 1964

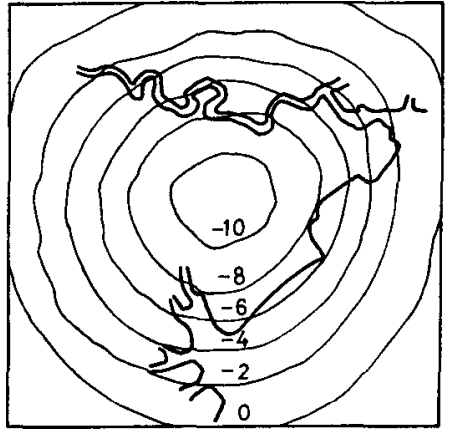

Feb : 1965

Fig. 8. Computed drawdown contours for homogeneous aquifer.

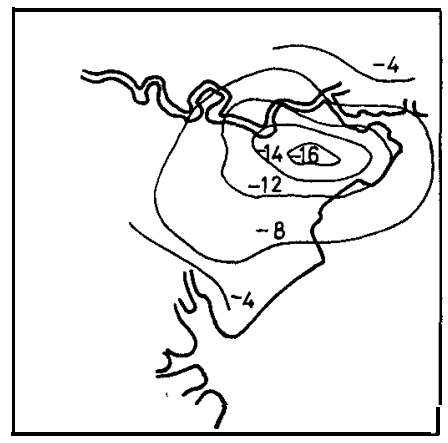

Sept : 1964

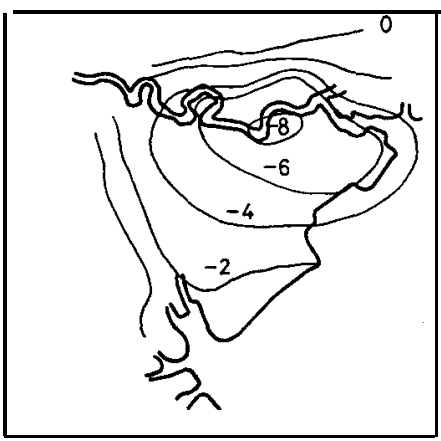

Feb : 1965

Fig. 9. Field contours. 
(2) The difference between these two can be attributed by assumed boundary conditions, non-homogeneity of the aquifer and replenishment from the confining layer.

The drawdowns were computed again by considering the non-homogeneity of the aquifer (transmissivity is variable with location), and the results obtained are shown in Fig. 10. By comparing it with actual data, computed values are still higher but their tendency is nearly the same.

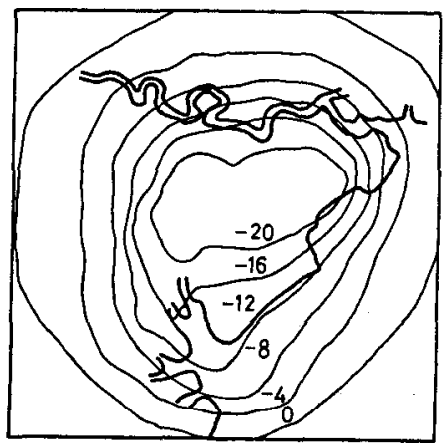

Sept : 1964

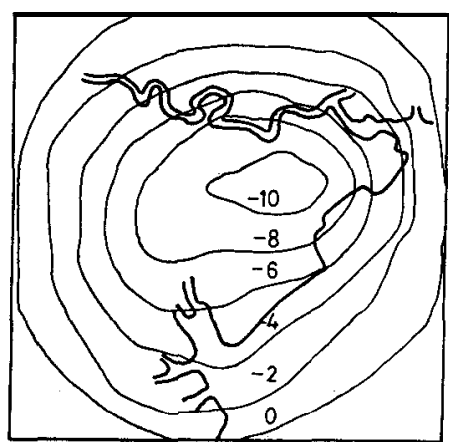

Feb : 1965

Fig. 10. Computed drawdown contours for non-homogeneous aquifer.

(3) Here the replenishment caused by vertical recharge from the confining layer is considered. As it is already mentioned that the storage of the confining layer is not taken into account, thus the water released from that layer due to squeeze is not neglected and recharge due to irrigation water is considered, that is the case when the steady state is reached in the confining layer.

The value of the vertical permeability was simulated within the limits of field data, the value of $1.0 \times 10^{-6} \mathrm{~cm} / \mathrm{sec}$ was found suitable for this data, the results obtained with this value are shown in Fig. 11. By comparing it with field data, the drawdown contours of Sept., 1964 are in good agreement with

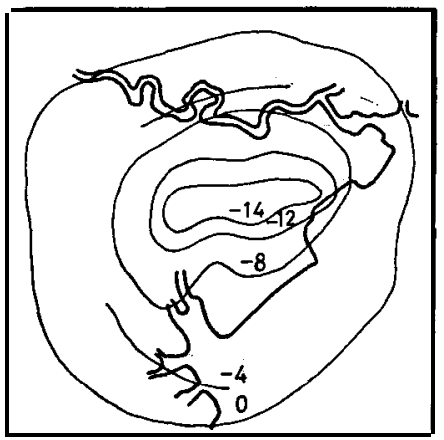

Sept : 1964

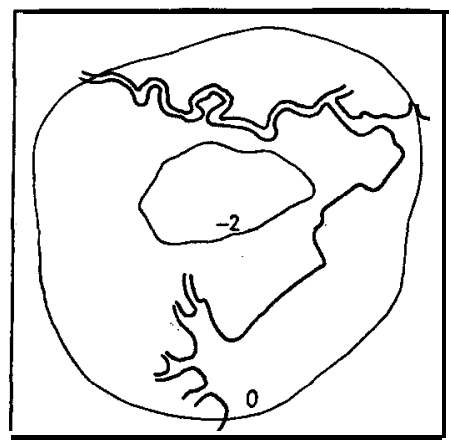

Feb : 1965

Fig. 11. Computed drawdown contours when vertical recharge is considered. 
field data but of Feb., 1965 are smaller. It was found also, that a little change in vertical hydraulic conductivity has great influence on the drawdowns. As shown in the previous results that computed drawdowns are smaller than field contours in Feb., 1965, so it was concluded that the replenishment of the main aquifer occurs in the irrigation period only. By assuming this the vertical recharge was cut off after Sept., 1964, and by doing this the results obtained are shown in Fig. 12 which match with field data.

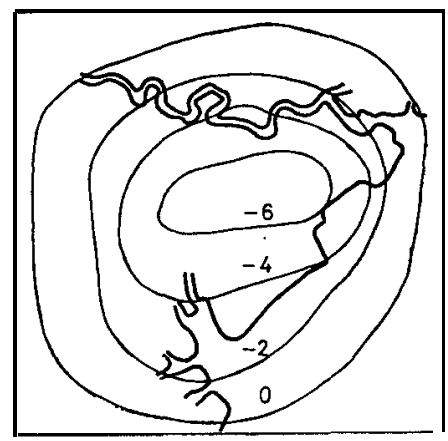

Feb : 1965

Fig. 12. Computed contours when vertical recharge is cut off, after Sept.

(4) When water is pumped from the aquifer it causes the decline of pressure head in the aquifer which results the entry of water from surrounding region, that can be called replenishment from the surrounding region or horizontal replenishment. The quantity of this recharge depends on the drawdown occurred in the aquifer and was computed in the following way;

a) This quantity as reported in the report of Agriculture and Forestry Authority of Saga Prefecture 1967 is computed by dividing the piezometric surface with flow lines (Fig. 13a) and then using the flow net equation.

$$
\mathrm{Q}=n_{f} / n_{d} T h \quad \mathrm{~m}^{3} / \mathrm{day}
$$

$\mathrm{Q}=$ Recharge quantity

$n_{d}=$ Number of equipotential lines

$\mathbf{h}=$ Average drawdown head $n_{f}=$ Number of flow lines

$\mathbf{T}=$ Transmissivity

b) Here Darcy's law is used to calculate the same quantity. In this method the drawdown heads on the nodal points next to fixed head nodal points (boundaries) are summed together and then quantity is calculated in the following way;

$$
\mathrm{Q}=K \cdot \Delta X \cdot b\left(h-h_{s}\right) / \Delta X=-K \cdot b \cdot h_{s}=-T \cdot h_{s}
$$

Recharge quantities obtained from both methods are compared in Fig. 13b, they vary in the same way but the later one (dotted line) is smaller than former (solid line), this difference may be due to the initial condition that was assumed zero before the aquifer was simulated. 


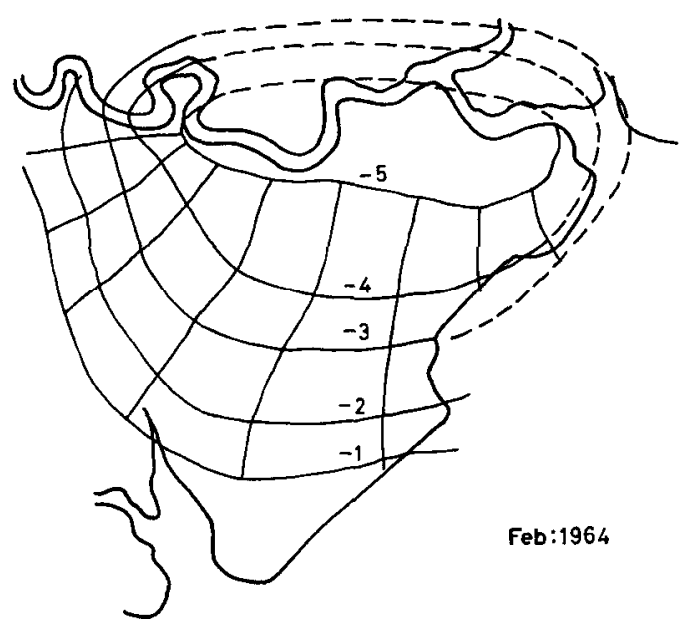

Fig. 13 (a). Flow net for horizontal recharge.

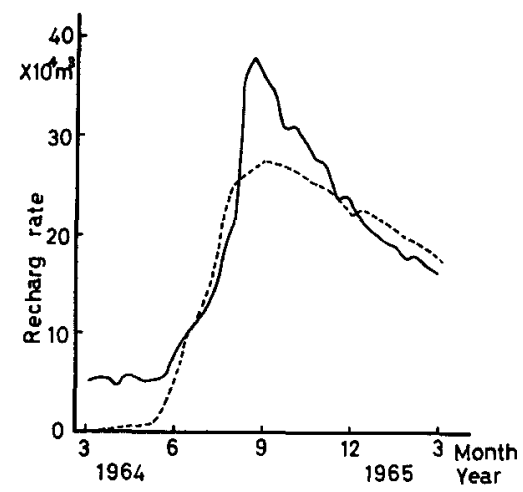

Fig. 13 (b). Recharge quantity.

(5) The replenishment of the aquifer is thus from vertical and horizontal recharge. In order to find out the percentage of each recharge, the horizontal and total recharge quantities are plotted in Fig. 14. The vertical recharge as men-

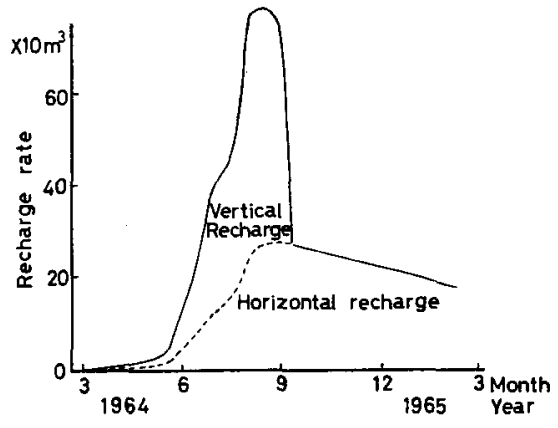

Fig. 14. Recharge quantity. 
tioned above was cut off after Sept., so there is only horizontal recharge after this month. From this figure it can be easily seen that the percentage of horizontal recharge is lesser than vertical recharge contribution. The horizontal recharge contributes $30 \%$ to $40 \%$ only and the remaining is from vertical recharge.

(6) The data of year 1966-1967 was analyzed with initial condition taken from the field data rather than zero drawdown as it was assumed in 1964-1965 data. It was found that the vertical permeability of $1.0 \times 10^{-6} \mathrm{~cm} / \mathrm{sec}$ does not give good results but of $1.25 \times 10^{-6} \mathrm{~cm} / \mathrm{sec}$ resulted better one. The actual and the computed drawdown contours are shown in Figs. 15 a and $15 \mathrm{~b}$ respectively and by comparison both of them are in good agreement. No doubt there is difference between them that may be due to assumed boundary conditions.

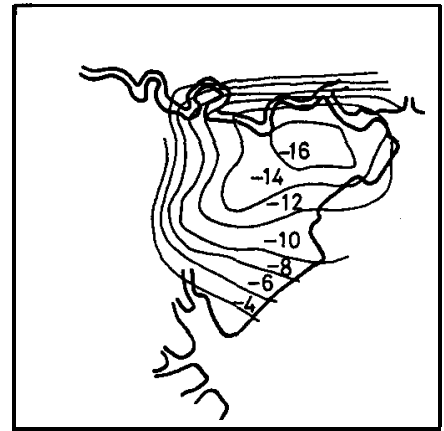

Sept 1966

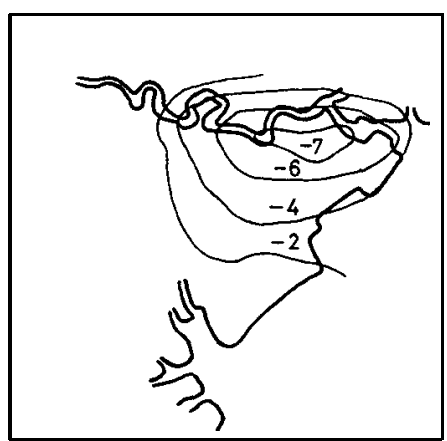

Mar 1967

Fig. 15 (a). Field contours.

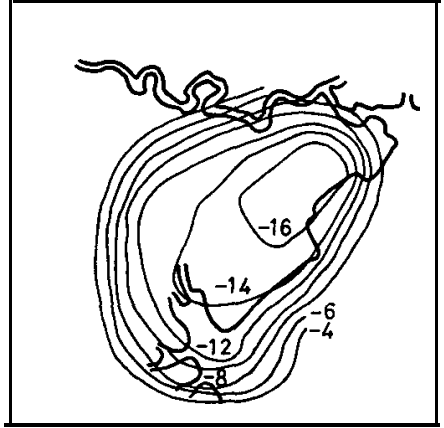

Sept : 1966

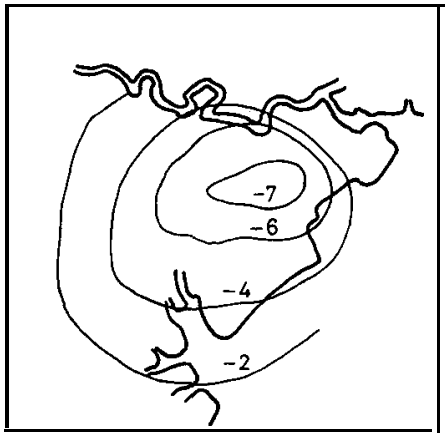

Mar : 1967

Fig. 15 (b). Computed contours.

(7) Fig. 16 shows the comparison of computed and measured drawdowns for year 1966-1967 at two points the locations of which are shown in Fig. 2. The field data was recorded two times a year so there are three values only, which are in good agreement with computed drawdowns. It can be easily concluded 

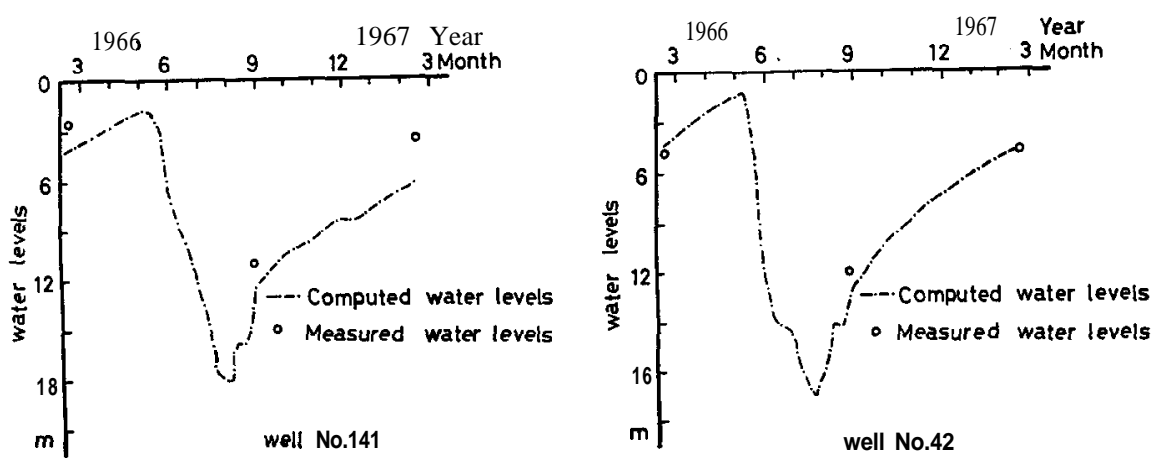

Fig. 16. Comparison of computed and field water levels at two points.

from this figure that the maximum drawdown occurs in the month of August, and water level is declining every year.

(8) Subsidence: It has been long understood that land subsidence is best analyzed with reference to the theory of consolidation, wherein it is well recognized that consolidation represents the response of a compressible porous medium to the changes in the fluid flow field operating with in it.

Schiffman et al. (1969) has classified theory of consolidations in the following way: a) Three dimensional theory, b) Pseudo three dimensional theory, c) Terzaghi's one dimensional theory. First two theories involve large number of factors so, practically a lot of work is required for their application, therefore there is seldom use of these in subsidence analysis. Up to this time all mathematical models of land subsidence have been direct application of Terzaghi's consolidation theory. This approach involves analytical solutions to the boundary value problem based on the one dimensional vertical equation of flow in which piezometric declination are specified as boundary conditions. Domenico and Mifflin (1965) provide an excellent review of this approach as it is applied to land subsidence.

$$
\frac{\partial^{2} u}{\partial z^{2}}=\frac{1}{C_{v}} \frac{\text { at } 4}{\mathrm{at}}
$$

$u=$ excess pore-water pressure, $C_{v}=$ co-efficient of consolidation. Total pressure in the porous medium is sum of neutral and effective pressure, where in the confined aquifer neutral pressure consists of hydrostatic and seepage pressure or transient pore-water pressure. Heads due to transient pore-water pressure causes flow in the aquifer that results in consolidation. When $u$ is solved as function of $\mathrm{z}$ and $t$, the equation describes the exact shape of family of curves (isochrones) showing the proportion of effective and neutral stresses in a confining bed from time $t=0$ when $\mathrm{u}$ begins declining to time $t=\infty$ when steady flow conditions are reestablished. For any given thickness, the greater the specific storage or the smaller the permeability, the greater the time required to establish a steady flow condition in the confining layer.

When water is pumped from main aquifer, it causes declination in that aquifer, 
owing to the lowering, effective and neutral pressure fractions acting through the overlying low permeability layer must seek a new equilibrium compatible with surrounding environment. The result is the transfer of neutral to effective, and thus increase in effective stress causes consolidation. Finally they derived the relation that the total volume of water removed from the prism of confining layer is equal to the product of specific storage and final effective pressure area, that can be determined from a depth-pressure diagram.

Here the mathematical simulation of subsidence is not carried out, but the relation of computed head decline, measured subsidence and thickness of confining layer is discussed in the following paragraphs:

a) In Figs 17a and 17b measured subsidence and computed drawdowns of 19661967 are plotted, at four points, from these figures it can be concluded that the time of the maximum subsidence and the maximum drawdown are coinciding with each other, but at some points maximum subsidence has been resulted later on, it is due to very little value of permeability of confining layer, and there is time lag of one to two months.

b) In Fig. 18 the subsidence measured in the field of months Aug. and Sept., thickness of clay and maximum computed drawdowns are plotted by dash and dot, broken and solid lines respectively. In this figure if drawdown contour is kept constant, then subsidence is more for thicker clay layer, for constant
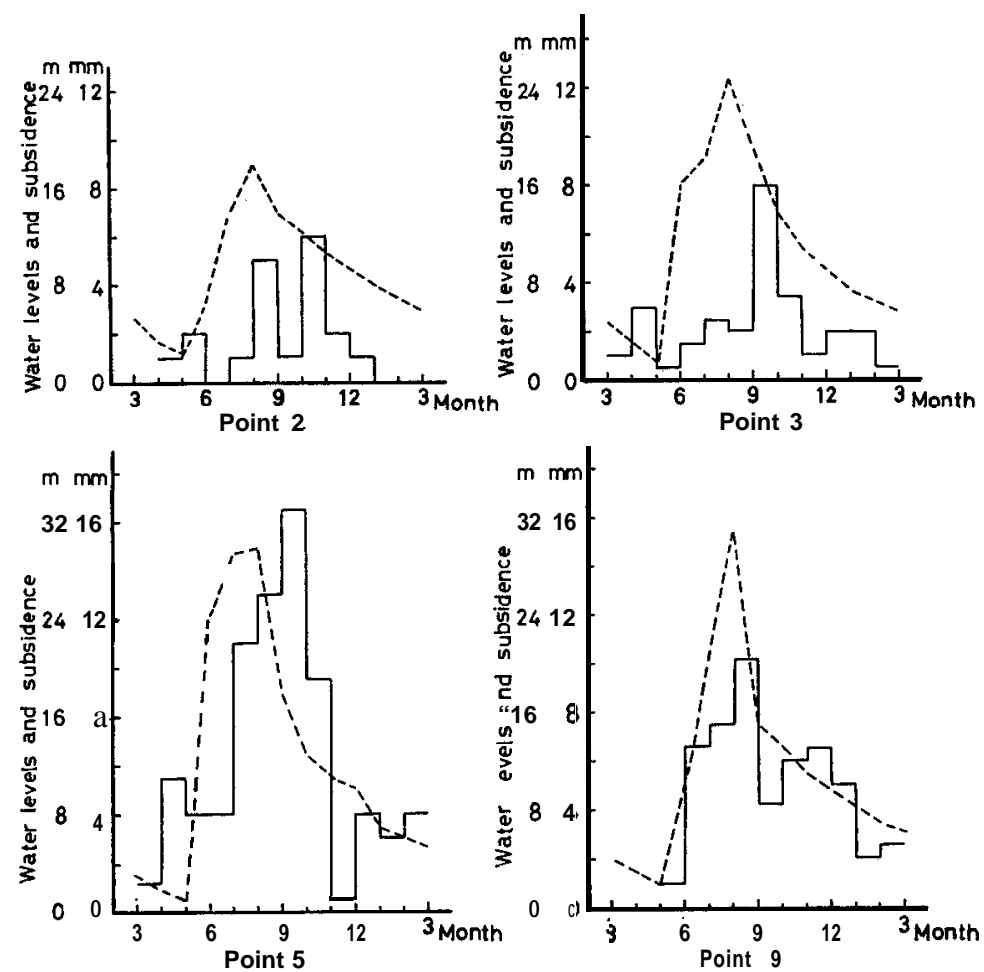

Fig. 17. Computed drawdowns and measured subsidence. 


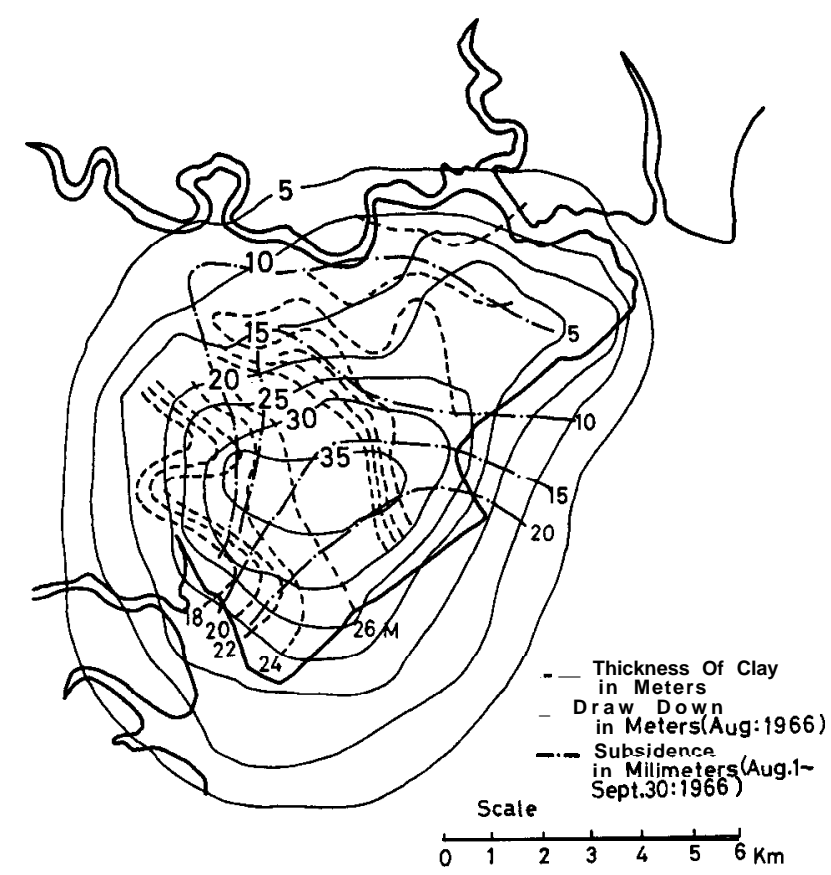

Fig. 18. Computed drawdowns, measured subsidence and thickness of clay.

thickness, the subsidence increases with increase in drawdown.

The main points of this research are summarized in the following paragraphs : (1) The numerical model of the Shiroishi area aquifer with appropriate boundary conditions have shown good results in comparison with field data. Therefore this model can be used to predict the future drawdowns for any pumping schedule. In this model it was found that the vertical recharge from confining layer should be considered, and further it was concluded that the replenishment of the aquifer due to vertical recharge occurs in irrigation period only, so it is necessary to cut off vertical recharge after the month of September.

(2) It was found that the maximum subsidence usually occurs one or two months later the maximum drawdown. Thus maximum drawdown and subsidence do not occur simultaneously, but there is lag of time between their occurrence.

(3) By plotting the contours of drawdown, subsidence and thickness of clay layer, it was concluded that for the same drawdown, subsidence is higher for thicker layer and for constant thickness of clay layer, subsidence is higher for higher drawdown.

(4) In this model the water released from confining layer due to squeeze was not considered, but in case of numerical analysis of subsidence it is necessary to consider the amount of water released from the storage of that layer.

The drawdown heads computed in this model can be used as time dependent boundary conditions for the numerical model to be used for numerical analysis of subsidence.

(5) In order to get better results it is necessary to consider the intervening 
aquicludes in the main aquifer and more reliable boundary conditions should be taken into account, and in this way more precise model can be achieved.

\section{ACKNOWLEDGEMENTS}

The comments and suggestions of Professor Dr. Kohei Tanaka and Associate Professor Dr. Masaharu Kuroda were very valuable for us. We are indebted to Mr. Akira Tanaka for his kind help otherwise. We had many discussions with him.

We wish to express our gratitude to Mr. Tamotsu Funakoshi for his help in drawing useful graphs.

\section{REFERENCES}

Agriculture and Forestry Authority of Saga Prefecture 1967 Shiroishi Heiya no Chikasui to Chikasui-chunyu Shiken (Groundwater and recharge test by the well method in the Shiroishi plain). Agriculture and Forestry Authority of Saga Prefecture, Saga (In Japanese).

Bredehoeft, J. D. and G. F. Pinder 1970 Digital analysis of areal flow in multiaquifer groundwater systems : A quasi three dimensional model. Water Resources Res., 6 (3) : 883-888

Cooper, H. H. 1966 The equation of groundwater flow in fixed and deforming coordinates. J. Geophys. Res., 71 (20) : 4785-4790

Domenico, P. A. and M. D. Mifflin 1965 Water from low permeability sediments and land subsidence. Water Resources Res., 1 (4) : 563-576

Gambolati, G. and R. A. Freeze 1973 Mathematical simulation of the subsidence of Venice. Water Resources Res., 9 (3) : 721-733

Jacob, C. E. 1940 The flow of water in an elastic artesian aquifer. Trans. Amer. Geophys. Union, 2: 574-586

McCarty, D. G. 1958 The use of high speed computers for predicting floodout patterns. Trans. Amer. Inst. Mining and Met. Engrs., 213 : 139-145

Peaceman, D. W. and H. H. Rachford 1955 The numerical solution of parabolic and elliptic differential equations. J. S c.Indust. Appl. Math., 3 (1) : 28-41

Schiffman, R. L., A. T. F. Chen and J. C. Jordan 1969 An analysis of consolidation theories, Proc. Amer. Soc. Civil. Eng., 95 (SMl) : 285-312

Tyson, H. N. and E. M. Weber 1961 Groundwater management for the nation's future-computer simulations of groundwater basins. Proc. Amer. Soc. Civil. Eng., 90 (H Y4): 59-77 